ISSN-PRINT 1794-9831 / E-ISSN 2322-7028

Vol. 18 No 3 / sep - dic, 2021 / Cúcuta, Colombia.

doi https://doi.org/10.22463/17949831.2850

Original

\title{
Proceso de trabajo de enfermería en la atención primaria de salud: estudio observacional
}

\author{
Work process of nursing in primary health care: observational study
}

Processo do trabalho da enfermagem na atenção primária à saúde: estudo observacional

\author{
Edna Johana Mondragón-Sánchez ${ }^{1 *}$ \\ Lina Karina Bernal-Ordoñez ${ }^{2}$ \\ Juliana Corpus-Quiguanás ${ }^{3}$
}

\section{Resumen}

Objetivo: Analizar el proceso de trabajo de los profesionales de enfermería en la APS, a partir del referencial teórico sobre proceso de trabajo en salud. Materiales y métodos: Se trata de un estudio cualitativo de tipo observacional. La investigación se efectuó en una Empresa Social del Estado que brinda servicios de APS en Colombia; la recolección de datos se llevó a cabo mediante la observación no participante. La muestra estuvo conformada por cuatro profesionales de la enfermería, seleccionadas intencionalmente, en función de sus roles y actividades desarrolladas en el contexto de la APS del municipio. Resultados: Mediante la observación se construyeron cuatro categorías temáticas, a saber: Proceso de atención; rol enfermero; comunicación y liderazgo con el equipo de trabajo; y, prácticas de educación para la salud. Conclusión: En el proceso de trabajo que realizan las enfermeras, predominan las tecnologías leves y leves-duras con sus saberes técnicos-científicos, por lo cual está presente el trabajo muerto que se torna vivo al establecer una relación recíproca enfermera/paciente.

Palabras clave: Atención primaria en salud; prevención primaria; enfermeros y enfermeras; rol profesional; práctica profesional.

\begin{abstract}
Objective: Analyze the work process of nursing professionals in the APS, from the referential theory about the process of work in health. Materials and methods: Qualitative study of observational approach. The research was carried out is a State-owned social enterprise that provides services of APS in Colombia; the recollection of data was made through non-participant observation. The sample was made by four nursing professionals, intentionally selected, in function of their roles and activities developed in the context of the APS of the municipality. Result: Throughout observation four thematic categories were built, namely: Process of assistance; nursing role, communication and leadership with the teamwork; and, practices of education for health. Conclusion: In the work process made by the nurses, mild technologies and mild-hard technologies predominate with their technical-scientific knowledge, whereby dead work is present and turns alive when establishing a reciprocal nurse/patient relationship
\end{abstract}

Keywords: Primary health care; primary prevention; nurses; professional role; professional practice.

\begin{abstract}
Autor de correspondencia*
${ }^{1 *}$ Enfermera, Magíster en enfermería. Docente Universidad del Quindío, Armenia, Colombia. Correo: ejmondragon@uniquindio.edu.co (i) 7950-2809

${ }^{2}$ Enfermera, Doctora en enfermería. Docente Fundación Universitaria del Área Andina, Pereira, Colombia. Correo: 1kbernal@uniquindio.edu.co(iD) 0000-0001-7486-0503

${ }^{3}$ Enfermera, Magíster en Gestión de Calidad en Salud. Docente Universidad del Quindío, Armenia, Colombia. Correo: jcorpus@uniquindio.edu.co (10) $\underline{0000-0002-3695-7379}$
\end{abstract}

Recibido: 9 febrero 2021

Aprobado: 22 junio 2021

\section{Para citar este artículo}

Mondragón-SanchezEJ, Bernal-Ordonez LK, Corpus-Quiguanás J. Proceso de trabajo de enfermería en la atención primaria de salud: estudio observacional. Rev. cienc. cuidad. 2021; 18(3):22-31. https:// doi.org/10.22463/17949831.2850

(C) Universidad Francisco de Paula Santander. Este es un artículo bajo la licencia CC-BY-NC-ND 


\section{Scientific Journal of Nursing}

\section{Resumo}

Objetivo: Analisar o processo de trabalho dos enfermeiros na APS, partindo do referente teórico sob o processo de trabalho em saúde. Materiais e Métodos: Trata-se de um estudo qualitativo observacional. A pesquisa foi desenvolvida numa instituição pública que presta serviços de APS na Colômbia. A coleta da informação realizou-se por observação não participante. A amostra foi integrada por quatro enfermeiros escolhidos intencionalmente, dadas as suas funções e atividades desenvolvidas no contexto da APS no município. Resultado: Construíram-se quatro categorias temáticas: processo de atendimento, papel do enfermeiro, comunicação e liderança da equipe de trabalho e, práticas de ensino para a saúde. Conclusão: No processo de trabalho realizado pela enfermagem, predominam as tecnologias leves e leves-duras com saberes técnicos e cientifico, pelo que se apresenta o trabalho morto tornando-se vivo ao estabelecer-se o relacionamento reciproco enfermeiro-paciente.

Palavras-Chave: Atenção primaria à saúde; prevenção primaria; enfermeiros e enfermeiras; papel profissional; pratica profissional

\section{Introducción}

La Atención Primaria de Salud (APS) según la definición dada en la Declaración de Alma-Ata de 1978, convocada por la Organización Mundial de la Salud (OMS), es la asistencia sanitaria fundamentada en métodos y tecnologías prácticos, científicamente fundados y socialmente aceptables, puesta al alcance de todas las personas, familias y comunidades, por medio de su plena participación. La APS es parte integrante del sistema de salud nacional, del cual, constituye la función principal y el núcleo central, así como, del desarrollo social y económico global de la comunidad (1).

Dicha definición enmarca la planeación y ejecución de acciones de diagnóstico, prevención, curación y rehabilitación. Para ello, implica un trabajo multi y transdisciplinar, de todos los actores del sistema que puedan influir en los determinantes sociales que afectan el bienestar del ser humano. En ese sentido, el rol que desempeña el profesional de enfermería en su trabajo, reviste gran interés para la implementación, gestión y planificación de los servicios de APS $(2,3)$.

Con relación al término "trabajo", Marx lo definió como el proceso que el hombre realiza conscientemente para transformar la naturaleza en elementos útiles para su vida. A su vez, el concepto de "proceso de trabajo" es la acción guiada por un fin, siendo este, la satisfacción de las necesidades humanas (4).
En el campo de la salud, el término "proceso de trabajo" fue discutido por primera vez por el autor Gonçalves (5), quien propone un análisis del "proceso de trabajo en salud" a partir de los siguientes elementos: objeto de trabajo que es todo aquello que va a ser transformado en la salud específicamente; los instrumentos corresponden a un conjunto de cosas que el trabajador incorpora entre él y el objeto de trabajo; la finalidad es la intención del proceso de trabajo; y agentes que hace referencia al sujeto o al conjunto de sujetos que ejecutan las acciones; al respecto se sugiere que estos sean vistos siempre de forma articulada, debido a que, un proceso de trabajo específico solo es dado por la relación recíproca entre esos elementos (5).

En el proceso de trabajo en salud siempre habrá un agente productor de actos de cuidado, (profesional de la salud) poseedor de conocimientos, equipamientos, herramientas y concepciones y un agente consumidor (paciente/usuario) poseedor también de concepciones, intenciones, necesidades y conocimientos (6).

A partir de esa perspectiva, Merhy (4) definió el trabajo vivo como el trabajo en acto, el trabajo creador y consciente; y el trabajo muerto hace referencia a los medios y productos finales del trabajo humano (herramientas, instrumentos y materiales). También dividió en tres grupos de herramientas tecnológicas, los medios y materiales que los trabajadores de la salud utilizan durante los encuentros con los pacientes, familias y comunidades, dependiendo de la finalidad y el sentido 


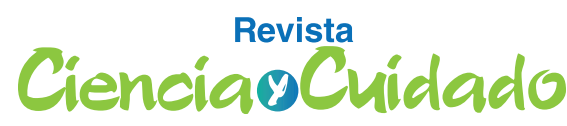

Scientific Journal of Nursing

que cada uno espera, los cuales corresponden a:

- Tecnologías duras, que son los equipamientos, instrumentos, procedimientos diagnósticos y terapéuticos; este grupo representa el trabajo muerto.

- Tecnologías leves-duras, son los saberes técnicos-científicos. Ese grupo también representa trabajo muerto puesto que se refieren a conocimientos definidos previamente, pero que se tornan vivos, cuando el trabajador actúa mediante su trabajo vivo en su encuentro con el paciente.

- Tecnologías leves, son las relaciones y encuentros entre trabajadores y pacientes, son las tecnologías que posibilitan la producción de vínculos a partir de la escucha, el acogimiento y la confianza, haciendo que el raciocinio clínico del trabajador de la salud sea más amplio y rico.

- Teniendo en cuenta lo anterior, es fundamental la transformación del actuar del equipo de salud; además hay que incorporar el modelo de salud familiar y comunitaria en los equipos básicos de salud, la organización de actividades no asistenciales, habilitación y adecuación de espacios físicos asumiendo un enfoque que tenga en cuenta acciones de promoción de la salud, prevención y detección temprana de la enfermedad e intervención de factores de riesgo de tipo familiar, ambiental e individual (7).

En ese sentido, el trabajo del profesional de enfermería abarca la salud de la población, la promoción de la salud, la prevención de las enfermedades, los cuidados de bienestar; convirtiéndose en el primer punto de contacto para la gestión de la enfermedad a lo largo de la vida. Para poder avanzar, es imprescindible que los profesionales de enfermería - en tanto figuras centrales en la prestación de los cuidados de la atención primaria de salud- participen, dirijan y coordinen los cuidados; pero además, que las competencias que desempeñen se consideren legítimas y esenciales en todos los sectores (8).

Partiendo de que la APS es la estrategia base e integradora del sistema de salud nacional, se considera importante identificar y analizar el proceso de trabajo de los enfermeros, puesto que, este es un tema emergente que ha sido poco estudiado y divulgado en Colombia. Como se demuestra en los resultados de revisión de la literatura realizada en el año 2019, se visualiza una laguna del conocimiento, con relación a la escasez de estudios publicados en los últimos cinco años, sobre la exploración y análisis de la actuación de los profesion-
Proceso de trabajo de enfermería en la atención primaria de salud: estudio observacional

ales de la enfermería en el contexto de la APS.

Los resultados de la presente investigación se revisten de gran importancia y de un aspecto práctico, ya que posibilitarán la promoción de discusiones y reflexiones relacionadas con la organización de los servicios de APS, a fin de contribuir a la actualización y fortalecimiento de los perfiles, competencias y prácticas de los enfermeros en el contexto de la APS. También, pueden coadyuvar para el apoyo en la planeación y realización de acciones y estrategias efectivas en el mejoramiento y mantenimiento de la salud de los colombianos, con el objeto de mejorar la resolutividad en los servicios y acciones de salud en el nivel primario de atención.

\section{Objetivo}

Analizar el proceso de trabajo de los profesionales de enfermería en la APS, a partir del referencial teórico sobre proceso de trabajo en salud.

\section{Materiales y Métodos}

Se trata de un estudio cualitativo de tipo observacional $(9,10,11)$. La observación es considerada como una técnica fundamental de la investigación cualitativa (9). Puede ser considerada como una técnica donde son recolectadas las impresiones y los registros acerca de un fenómeno determinado, a través de un contacto directo con las personas observadas o de instrumentos que auxilian el proceso de observación, con la finalidad de recoger datos suficientes para la realización de la investigación (10).

La investigación se desarrolló en una Empresa Social del Estado que brinda servicios de APS en un municipio de Colombia, durante el periodo comprendido entre enero yfebrero del año 2020. La técnica de recolección de datos utilizada fue la observación no participante, en la que el observador entra en contacto con el sujeto de estudio, observando de forma imparcial, sin prejuicios y sin integrarse en sus actividades cotidianas (11). Los criterios de inclusión de la muestra, fueron el ser profesionales en enfermería en el área de APS, además de haber aceptado participar en la investigación.

El universo de la población está conformado por 13 enfermeras que actúan en la red de APS del municipio seleccionado; sin embargo, en el momento de recolección de los datos, dos enfermeras se encontraban en vacaciones, una en licencia de maternidad y seis no aceptaron ser observadas en la cotidianidad de su trabajo, por tanto, la presente investigación contó con la participación de cuatro enfermeras, con los siguientes cargos: una enfermera coordinadora general de APS 
Cienciagechidado

Scientific Journal of Nursing

del municipio, dos coordinadoras de centros de salud que a su vez realizan actividades asistenciales y una enfermera asistencial.

Quienes se desempeñan como coordinadoras, realizan actividades de: solicitud de insumos de trabajo (materiales de aseo y biomédicos), realización de informes, comunicación al equipo de trabajo, liderazgo colectivo, cumplimento de metas y estrategias propuestas por los entes nacionales con relación a APS. Dichos roles las coloca en una constante tensión entre la administración/gestión de los servicios de salud y la prestación de la consulta y el cuidado directo de enfermería a individuos, familias y comunidades.

La observación no participante se efectuó durante dos jornadas laborales en un período ininterrumpido de 8 horas con cada enfermera participante. El contacto con cada participante se hizo previamente vía llamada telefónica, desde donde se confirmó la intención de participar en la investigación, y se acordó el día y la hora en que se haría la recolección de la información. Por otra parte, los datos de contacto fueron suministrados por la institución participante, la cual, previamente avaló la realización del estudio. Durante esta etapa participó un solo observador previamente capacitado en la técnica, utilizando un instrumento guía que condujo el proceso de observación. Dicho instrumento fue construido por las tres investigadoras y validado por un experto enfermero con amplia trayectoria y experiencia en la APS de otro municipio de Colombia. El instrumento guía comprendió datos de identificación del sujeto de estudio, fecha, hora de inicio y de terminación de la observación, y elementos alusivos a la descripción del proceso de trabajo, como horarios, duración, acciones realizadas y el contexto en que las mismas se desarrollaron.
Para el registro de las observaciones se utilizó un diario de campo, estos datos fueron sistematizados en un libro de Excel por parte de la investigadora que realizó el proceso de observación. En el análisis de los datos participaron las tres investigadoras autoras del presente estudio, se utilizó la metodología de análisis de contenido, en la modalidad categorial temática propuesta por Bardin $(12,13,14)$. El análisis de los datos se realizó a partir de la teoría de "Proceso de Trabajo en salud" (15).

La presente investigación fue avalada por el Comité de Ética de la Facultad de Ciencias de la Salud de la Universidad del Quindío con el Acta $\mathrm{N}^{\circ} 35$ 05-10-2018. Se respetaron los principios éticos de beneficencia, no maleficencia, justicia y respeto a la autonomía. Se tuvo en cuenta la Resolución 008430 de 1993, para la calificación del riesgo de la presente investigación, la libertad de expresión, la confidencialidad de la información y la identificación de los participantes, respeto al aceptar participar o no en la investigación y la devolución de los resultados a la institución y participantes, según la Ley 1581 d 2012 y el decreto 1377 de 2013. Todas las participantes firmaron el consentimiento informado.

Las investigadoras hacen parte del Grupo de Investigación de Atención Primaria de Salud (GIAPS), reconocido por MinCiencias - Colombia.

\section{Resultados}

La caracterización de las cuatro enfermeras que fueron observadas, con relación a la edad, tiempo de experiencia en APS y postgrados, se encuentra ilustrada en la tabla 1.

Tabla 1. Relación de edad, tiempo de experiencia en APS y postgrados de enfermeras.

\begin{tabular}{ccccc}
\hline Enfermera & Sexo & Edad & $\begin{array}{c}\text { Tiempo de Experien- } \\
\text { cia en APS }\end{array}$ & $\begin{array}{c}\text { Estudios Pos graduales } \\
\text { E1 }\end{array}$ \\
Femenino & 57 años & 32 años & No posee \\
E2 & Femenino & 32 años & 9 años & $\begin{array}{c}\text { Especialista en epidemi- } \\
\text { ología }\end{array}$ \\
E4 & Femenino & 40 años & 3 años & No posee \\
& Femenino & 24 años & 2 años & No posee \\
\hline
\end{tabular}

Fuente: Elaborado por las Autoras. 


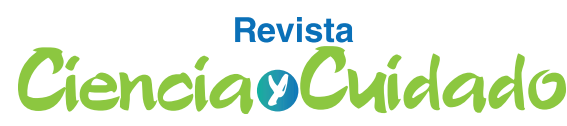

Scientific Journal of Nursing

A continuación, se identifican los elementos que componen el proceso de trabajo en la APS, de acuerdo con el referencial teórico propuesto, en referencia a: los agentes, el objeto, los instrumentos y la finalidad (5).

Con relación a los agentes del proceso de trabajo en la APS, en el presente estudio se identificaron como agentes, las cuatro enfermeras participantes, pues ejercen un rol de liderazgo en los procesos de APS del municipio, en lo relacionado con la coordinación del equipo de trabajo que está compuesto por: médico general,

interno de medicina, auxiliares de enfermería, auxiliar de facturación, odontólogo e higienista oral.

Acerca del objeto de trabajo en la APS, se identificaron las necesidades en la salud de individuos, familias y comunidades que se atienden en los centros de salud en donde las enfermeras actúan, planeando, implementando y evaluando intervenciones de cuidado, cuya finalidad es la resolución de dichas necesidades y el mejoramiento de la calidad de vida de las personas.

También, de forma general, se observó que en el cuidado directo que las enfermeras ofertan durante la consulta de enfermería, tuvieron en cuenta las necesidades particulares y específicas de cada paciente y familia; de igual forma, la relación enfermera-paciente/familia se establece por medio de la empatía y la simpatía, manteniendo una comunicación asertiva con su equipo de trabajo.

Del mismo modo, se observó que los principales instrumentos y herramientas que utilizan las enfermeras en su quehacer cotidiano, están relacionados con el diligenciamiento de formularios y fichas de valoración durante la consulta; con el uso de equipos biomédicos presentes en los consultorios donde atienden dichos profesionales, los cuales representan instrumentos materiales. En cuanto a los instrumentos no materiales que utilizan las enfermeras en su cotidianidad, se identificaron con los conocimientos técnico-científicos propios de la disciplina y sobre APS, además, de las habilidades técnicas para brindar la atención y el cuidado respectivo.

Para una mejor comprensión del análisis de los datos, se construyeron cuatro categorías analíticas, que emergieron de los mismos, a saber: Proceso de atención; Rol del enfermero; comunicación y liderazgo con el equipo de trabajo; y, prácticas de educación para la salud. A continuación, se describen las actividades observadas y descritas en el diario de campo:

Proceso de Atención

Se observó que las enfermeras llamaron por su nom-
Proceso de trabajo de enfermería en la atención primaria de salud: estudio observacional

bre a los pacientes, mantuvieron contacto visual con ellos durante las consultas, y ayudaron al paciente a reconocerse a sí mismo como alguien de valor: "Martha, recuerda que tienes que cuidarte y tuvales mucho más que 2000 pesos" (nota del Diario de campo).

También durante la interacción con el usuario, se advirtió que las enfermeras utilizan herramientas como la historia clínica y las escalas de valoración sistematizados; además disponen de equipos biomédicos que les permiten conocer con mayor profundidad el estado de salud del paciente.

Educaron a los pacientes sobre la importancia del autocuidado "Recuerda que debes venir en un mes por el resultado de la citología, y debes de practicar el autoexamen de seno como acabo de explicarte" (nota del Diario de campo).

Durante ninguna de las consultas se evidenció que las enfermeras indagaran sobre las creencias religiosas, culturales o espirituales de los pacientes y familias atendidas.

Desde otro punto de vista, las enfermeras expresaron que tenían que enfrentarse diariamente durante las consultas, a diversos desafíos relacionados con la salud de sus pacientes, por lo que "utilizar un modelo holístico de atención es lo ideal" (nota del Diario de campo). Sin embargo, debido a que el tiempo de la consulta es limitado y en ocasiones insuficiente para desarrollar una consulta de enfermería completa y holística, una de las enfermeras manifestó durante el proceso de observación, que se sentía impotente por no poder brindar un cuidado holístico a sus pacientes.

\section{Rol Enfermero}

Se observó en las cuatro enfermeras la presencia del rol independiente e interdependiente en la práctica de APS. Con relación al rol independiente las profesionales construyen y se esfuerzan por mantener un vínculo con los pacientes, familias y/o comunidad; además, se muestran como principales responsables en la evaluación y control de la atención brindada. El rol interdependiente se visualizó en las actividades y funciones que realizan con los demás miembros del equipo de salud, donde realizan actividades de planeación, implementación y evaluación de las acciones en salud, en conjunto con el equipo interdisciplinario sobre el cuidado integral de las personas, familias y comunidades.

El rol de las cuatro enfermeras está representado en las diferentes acciones que dan solución a los problemas de salud, evidenciado en la atención directa o consul- 
ta de enfermería en: asesoría de planificación familiar, realización de la toma de muestra de citología cérvico uterina, asesoría Pre y Pos - test de HIV, colocación y retiro de dispositivo intrauterino (DIU), atención a la primera infancia y adolescencia; analizando la información recogida durante la valoración, para diseñar y proponer acciones de cuidado de la enfermería, las cuales se registran en las historias clínicas.

\section{Comunicación y liderazgo con el equipo de trabajo}

Durante la observación se evidenciaron algunas habilidades del liderazgo en las cuatro enfermeras, como: conocimiento técnico-científico, experiencia profesional, confianza, seguridad, autonomía, habilidades para el trabajo en equipo, capacidad para resolver problemas, autodesarrollo, buenas relaciones interpersonales, respeto a los miembros del equipo de salud, habilidades de escucha y diálogo: "Lo que hace que una persona sea un líder es su capacidad de escuchar, de tener iniciativa y de preparación y eso es lo que yo intento realizar con mi equipo de trabajo" (Nota del Diario de campo). "Nuestra formación contribuye mucho para que seamos líderes, desde la atención de enfermería, planificación, análisis de problemas, clasificación de prioridades y solución de problemas" (Nota del Diario de campo).

Del mismo modo se observó que en la práctica las enfermeras se comunican de una manera amable y respetuosa con su equipo de trabajo, pacientes, familias y comunidad: "Augusto, ¿usted me podría sacar una copia de estos resultados que necesito, por favor?" (Nota del Diario de campo).

No obstante, en la práctica de una enfermera, se observó una comunicación impersonal, no se evidenció contacto visual o comunicación directa entre ella y su equipo de trabajo; la comunicación fue mediada predominantemente por el uso del celular personal a través de llamada o mensaje en WhatsApp; esta enfermera permaneció dentro de su consultorio con la puerta cerrada. Esta situación se podría atribuir a que la ubicación del consultorio físico de la enfermera, se encuentra distante de la recepción y sala de espera; también, estas acciones pueden estar asociadas a los cambios de conducta al sentir que está siendo observada.

\section{Prácticas de Educación para la Salud}

Se observó que es el momento de la consulta de enfermería donde se realizan acciones de educación para la salud con los pacientes, familias y/o comunidad; es allí donde se detectan las necesidades educacionales y se elaboran acciones de educación para la salud, en la búsqueda de la promoción de la salud y prevención de la enfermedad, como por ejemplo: aclaraciones sobre la condición de salud o patología, incentivo de los hábitos de vida saludable, prevención de Infecciones de Transmisión Sexual (ITS), alimentación sana, prevención cáncer de seno y cuello uterino.

\section{Discusión}

La discusión de los resultados, presentada a continuación, se fundamenta en la caracterización de las actividades desarrolladas por las enfermeras que actúan en la Red de APS en un municipio de Colombia. De modo general, la discusión presenta la constitución de los procesos de trabajo de enfermeras en la APS, en las dimensiones de administrar, asistir/cuidar, educar y liderar, presentando sus elementos e interrelaciones entre ellos, dando a conocer que el trabajo de enfermería en la APS es complejo y multifacético, y que por tanto, requiere de un conjunto de conocimientos, habilidades y actitudes que se articulan de manera propia para producir transformaciones y contribuciones sociales.

Conviene subrayar que los profesionales enfermería ejecutan su "Acto de trabajo" ["Acto de cuidado] con un saber que les permite aprehender "su objeto social" [el cuidado de la salud de los sujetos de cuidado: individuos, familias y comunidades] a partir de necesidades humanas que se identifican durante la valoración bio-psico-social-emocional del o de los sujetos de cuidado. Para emitir un juicio y diagnóstico a fin de plantear un proceso de enfermería eficaz en APS, el profesional de enfermería debe lograr captar el objeto social, o el sentido de su "Acto de cuidado" y para esto, despliega toda una batería de tecnologías materiales y no materiales.

Con relación a lo anterior, se pudo observar que las enfermeras reconocen en su prácticas, la realización de procesos de atención de enfermería con un abordaje holístico para poder dar respuesta y contribuir en la resolución de las necesidades de individuos, familias y comunidad. No obstante, manifestaron que diferentes situaciones relacionadas propiamente con el sistema de salud, dificultan ese proceso, lo que provoca el desarrollo de procesos de atención de enfermería con una lógica predominantemente biomédica, pautada en la estructura funcionalista. Diferente a lo que propone Jean Watson (16), quien considera los procesos de atención de enfermería como un abordaje holístico y humanístico, que se centra en la atención del paciente reconociendo la unidad entre cuerpo, mente, espíritu y emoción. En efecto la realización de un proceso de atención de la enfermería no solo permite desarrollar los patrones del 


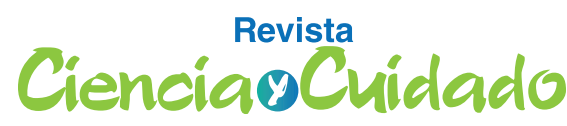

Scientific Journal of Nursing

conocimiento en ésta especialidad, sino también generar una relación más íntima y personal con el ser humano que se cuidada, brindándole la posibilidad de que sea protagonista sobre su propia salud, tomando decisiones que mejoren su calidad de vida $(17,18,19)$. Visto desde esta perspectiva, los instrumentos de trabajo deberían ser comprendidos como ese "poner en juego, llevar a la práctica y desplegar el proceso de enfermería" con todos sus patrones de conocimiento: empírico, estético, ético, personal. Es en esta dimensión tecnológica del trabajo de la enfermería, lo que le permite responder de manera satisfactoria a las necesidades humanas específicas de cada situación de cuidado que se identifique y reinterpretar su "Acto de trabajo" ["Acto de cuidado] en las APS, articulando lo social-concreto observado en los contextos laborales, con las posibilidades científicas de la disciplina.

De acuerdo con Irvine, Sidani, y McGillis Hall (20), quienes han desarrollado el Modelo de Eficacia de la Función de Enfermería, éste se puede utilizar para guiar la evaluación de la contribución de las enfermeras; en ese contexto, se afirma que el papel de la enfermería en el modelo conceptual se basa en el modelo de estructura-proceso-resultado del modelo de atención en salud desarrollado por Donabedian, además se adapta para medir la calidad del rol de la enfermería.

De igual forma, con relación al Rol de Enfermero se observó en la práctica de las enfermeras el desarrollo del rol independiente y el interdependiente, resultado que también fue encontrado en el estudio de Tuesca et al., (7) y en la OPS en el 2018 (21), donde se refleja que el rol de los enfermeros está en relación directa con los significados, las construcciones teóricas, el enfoque de su trabajo y su papel en escenarios de actuación de la APS.

En función del planteamiento anterior, el rol independiente de las enfermeras observadas, está presente en las actividades propias de la disciplina, siendo estas funciones y responsabilidades únicas del profesional de la enfermería, tales como: la valoración del paciente, la toma de decisiones sobre el cuidado del mismo, la planeación e implementación de las intervenciones de enfermería; igualmente, están presentes actividades de seguimiento y evaluación de dichas intervenciones. Hallazgos similares se encontraron en el estudio de Bryant et al. (22), quienes identificaron en los enfermeros estudiados, actividades del rol independiente como la exploración física de la enfermería, la planificación y la organización de actividades, planeación, implementación y evaluación de los planes de cuidado para los pacientes; es decir, planear, organizar y dirigir todo lo concerniente al recurso humano propio de la
Proceso de trabajo de enfermería en la atención primaria de salud: estudio observacional

enfermería $(20,23,24)$.

En cuanto al rol interdependiente (25) se evidenciaron funciones y responsabilidades planificadas y ejecutadas en conjunto con otros miembros del equipo de salud multidisciplinario. Resultados similares se encontraron en una Revisión Integrativa en el cual los autores concluyeron que el rol interdependiente de la enfermería intenta describir cómo ha de relacionarse la enfermera con los demás integrantes del equipo de salud, de tal forma que puede interactuar con la mayor exactitud de la información, como establecer una comunicación y coordinación más efectiva y dar cumplimiento a los objetivos y/o metas propuestas (26). Además, se resalta la importancia en un estudio interpretativo fenomenológico realizado en Colombia, en el cual los autores concluyeron que los profesionales de la enfermería se involucran, pero dependen parcialmente de las funciones de otros proveedores del cuidado en salud, para ejecutar sus propias actividades (27).

En otros estudios se concluyó que el rol del profesional de enfermería no es muy claro para los administradores de servicios de salud; tampoco está claro el trabajo de los equipos de salud que no está articulado con los principios de la APS; además, se demostró que las relaciones entre enfermeros y administradores de los servicios de salud fueron deficientes y sentían falta de apoyo por parte de los mismos (2).

Conviene subrayar que se identificaron en las enfermeras observadas, algunas habilidades de Comunicación y liderazgo con el equipo de trabajo, como: amplía experiencia de actuación en APS, apropiación de conocimientos científicos, seguridad, confianza, capacidad de escucha, relaciones interpersonales respetuosas y empáticas, entre otras. Estos hallazgos coinciden con los encontrados en un estudio que pretendió realizar un análisis teórico para describir los constructos básicos de la psicología positiva, relacionados con elementos como el compromiso, la pasión en el trabajo, la centralidad y el significado del trabajo, que podrían actuar como factores protectores en la profesión de la la enfermería, donde se afirma que "Sin comunicación no puede haber liderazgo" (28); es decir, que una de las principales cualidades de un líder es la capacidad de comunicar e influenciar a las personas (29) .

Agencias internacionales y asociaciones gremiales abogan para que se integre el profesional de la enfermería tanto en la planificación y gestión de la APS, como en el liderazgo y comunicación, como estrategias a desarrollar en el perfil del enfermero $(30,31)$. Por tanto, la actuación de la enfermería debe estar orientada a la conquista de la promoción de la salud con liderazgo y 
comunicación, según los principios de Alma Ata que estimula las acciones de prevención, la recuperación y la rehabilitación de los individuos, teniendo en cuenta sus necesidades, su entorno y el ciclo vital $(7,32)$.

Con respecto a las Prácticas de Educación para la Salud se observaron durante la realización de los atendimientos o consultas de enfermería; además, se pudo evidenciar que la educación en salud ayudó a reducir la ansiedad del paciente y también, generó una mayor confianza y vínculo entre el paciente y la enfermera. Resultados similares se encontraron en un estudio donde se afirma que es importante que las enfermeras desarrollen habilidades para diagnosticar la ansiedad y tratarla, con el fin de disminuir el impacto negativo en los pacientes, permitiendo que asuman una actitud de mayor compromiso en la toma de decisiones relacionadas a su salud $(22,25,33,34)$.

Lo expresado permite concluir que "el proceso de trabajo" en las APS posibilita escenarios de trabajo vivo, creativos y no estandarizados; son espacios de encuentro de singularidades, de subjetividades en acción, de diálogo, de intercambio, en los que predominan las tecnologías leves y leves duras, relacionales, como referentes para orientar el uso de otras tecnologías. El contexto de la APS permite reinventar el saber-hacer profesional de la enfermería día a día. Por tanto, en este espacio social, el planteamiento del proceso de enfermería es autónomo y se ejecuta con alto grado de incertidumbre, y es precisamente esta característica de flexibilidad la que torna viable el desarrollo de estrategias que posibiliten la construcción de nuevos valores, comprensiones y relaciones para hacer que el proceso del trabajo del enfermero dé un "sentido social" que priorice las necesidades humanas de la salud de los sujetos de cuidado.

\section{Conclusiones}

- La observación de las prácticas de las enfermeras permitió identificar todos los componentes del proceso de trabajo (objeto, agentes, instrumentos y finalidad).
- Predomina el uso de herramientas / instrumentos enmarcados en las tecnologías leve y leve-duras, lo que se relaciona con el tiempo de atención a los usuarios, así como de la estructura y los lineamientos del sistema de salud en el que se enmarca dicha atención.

- El rol que desempeñan las enfermeras evidencia que el profesional de enfermería es un líder en el proceso de atención primaria y a su vez articula el trabajo con los demás integrantes del equipo de salud y se comporta como un vínculo fundamental entre el usuario y el sistema de salud.

- Los profesionales de la enfermería poseen conocimientos suficientes para brindar una educación para la salud pertinente, fortaleciendo el proceso de aprendizaje con una mayor interacción con el usuario, a fin de que el aprendizaje sea significativo.

- Los enfermeros deben fomentar estrategias que les permitan una mayor valoración y consiguiente un mayor uso de tecnologías leves durante la atención, logrando una mejor relación y vínculo con los pacientes, familias y comunidades, a través de la confianza, la escucha y el acogimiento, entre otros, para así fortalecer el proceso de atención de la enfermería fundamentado en una visión holística del ser humano.

Agradecimientos: A la institución E.S.E. Red Salud de Armenia por permitirnos realizar la recolección de datos de la presente investigación con profesionales que hacen parte de su equipo de trabajo.

Divulgación: Las autoras no informan ningún conflicto de intereses, y son las únicas responsables del contenido y la redacción de este artículo.

Financiamiento: Las autoras declararon haber recibido apoyo financiero para la investigación, autoría y / o publicación de este artículo. Este trabajo fue apoyado por la Vicerrectoría de Investigaciones de la Universidad del Quindío

\section{Referencias bibliográficas}

1. Organización Panamericana de la Salud. (OPS). Conferencia Internacional sobre Atención Primaria de Salud, Alma-Ata, URSS, 6-12 de septiembre de 1978. [Internet] 2012. Disponible en: https://www.paho.org/hq/ dmdocuments/2012/Alma-Ata-1978Declaracion.pdf

2. Poghosyan L, Shang J, Liu J, Poghosyan H, Liu N, Berkowitz B. Nurse practitioners as primary care 
providers. Healt Care Management Review. 2015; 40(1):46-55. Disponible en: https://doi.org/10.1097/ HMR.0000000000000010

3. Bryant-Lukosius D, Valaitis R, Martin-Misener R, Donald F, Peña LM, Brousseau L. Advanced practice nursing: A strategy for achieving universal health coverage and universal access to health. Revista Latino-Americana de Enfermagem [Internet]. 2017 Jan 30 [cited 2021 Jun 10]; 25: e2826. Disponible en: https://doi. org/10.1590/1518-8345.1677.2826

4. Merhy EE. Em busca do tempo perdido: a micropolítica do trabalho vivo em saúde. Agir em Saúde: um desafio para o público. São Paulo: Hucitec. 2008; 71-112. Disponible en: https://digitalrepository.unm.edu/ lasm pt/145

5. Mendes-Gonçalves RB. Tecnología y Organización de las Prácticas de Salud. Características Tecnológicas del Proceso de Trabajo en la Red Estatal de San Pablo. 2008. Disponible en: https://digitalrepository.unm.edu/ $\underline{\text { lasm es/10 }}$

6. Macruz-Feuerwerker LC. Micropolítica e Saúde: produção do cuidado, gestão e formação. Coleção Micropolítica do Trabalho e o Cuidado em Saúde. Publisher: Editora Rede Unida.; 2008. Disponible en: https://doi. org/10.13140/2.1.1424.0646

7. Tuesca-Molina R, Segura-Barrios I, Bruno-Rubio V, Bustamante-Llinás M, Jiménez-Hamburgen A, Maldonado-Mendoza L. Atención Primaria en Salud. Una mirada desde los profesionales de enfermería: Barreras, conocimientos y actividades. Barranquilla-Colombia. Revista Científica Salud Uninorte, 2017; 31(2):295-308.

8. Gonzalez-Escobar DS. Enfermería y la atención primaria de salud. Revista Ciencia y Cuidado [Internet]. 2019 Apr [cited 2021 Jun 10]; 16(1):5-7. Disponible en: https://doi.org/10.22463/17949831.1586

9. Kirk J, Miller M. Reliability and Validity in Qualitative Research. SAGE Publications, Inc.; 2012. Disponible en: https://us.sagepub.com/en-us/nam/reliability-and-validity-in-qualitative-research/book1797

10. De Moura ML, Ferreira MC, Paine PA. Manual de elaboração de projetos de pesquisa. EdUERJ; 1998.

11. De-Andrade-Marconi M, Lakatos EM. Fundamentos de Metodologia Científica [Internet]. 5th ed. São Paulo: EDITORA ATLAS S.A.; 2003 [cited 2020 Dic 10]. Available from: https://docente.ifrn.edu.br/olivianeta/ disciplinas/copy of historia-i/historia-ii/china-e-india

12. Vergara SC. Réplica 2 - análise de conteúdo como técnica de análise de dados qualitativos no campo da administração: potencial e desafios. Revista de Administração Contemporânea. 2011; 15(4):761-765. Disponible en: https://doi.org/10.1590/s1415-65552011000400012

13. Bardin L. Análise de conteúdo (L. de A. Rego \& A. Pinheiro, Trads.). Lisboa: Edições 70, 2016.

14. Abela JA. Las técnicas de Análisis de Contenido: Una revisión actualizada. 2018. Disponible en: http:// mastor.cl/blog/wp-content/uploads/2018/02/Andreu.-analisis-de-contenido.-34-pags-pdf.pdf

15. Merhy EE, Feuerwerker LCM. Novo olhar sobre as tecnologias de saúde: uma necessidade contemporânea. 2009. In Mandarino ACS; Gomberg E. (org.) Novas tecnologias e saúde. Salvador: EdUFBa.

16. Guerrero-Ramírez R, Meneses-La Riva ME, De La Cruz-Ruiz M. Cuidado humanizado de enfermería según la teoría de Jean Watson, servicio de medicina del Hospital Daniel Alcides Carrión. Lima- Callao, 2015. RENH [Internet]. 2017 [citado 9jun.2021]; 9(2):133-142. Disponible en: https://revistas.upch.edu.pe/index.php/ $\underline{\text { RENH/article/view/3017 }}$

17. Santos BM, Silva RM, Pereira ER, Joaquim FL, Pinto T. percepção dos estudantes de Enfermagem sobre o cuidado humanizado: revisão integrativa. Revista Brasileira de Enfermagem. [Internet] 2018; 71(6): $2965-2973$. Disponible en: https://doi.org/10.1590/0034-7167-2017-0845

18. Federico L. Modelos y Teorías en la ciencia del cuidado. Aclaraciones epistemológicas. Cultura de los Cuidados (Edición digital). 2020; 24(56). Disponible en: https://rua.ua.es/dspace/bitstream/10045/106024/1/ CultCuid56-300-314.pdf

19. Mijangos-Fuentes KI. El paradigma holístico de la enfermería. Revista Salud y Administración. 2014; 1(2):17-22. Disponible en: http://www.unsis.edu.mx/revista/doc/vollnum2/A3 Paradigma_Holistico.pdf

20. Irvine D, Sidani S, Hall LM. Linking outcomes to nurses' roles in health care. Nurs Econ. 1998; 16(2):58-

87.

21. Organización Panamericana de la Salud. (OPS). Ampliación del rol de las en enfermería enfermeras y enfermeros en América Latina en la atención primaria de salud y el Caribe. In Organización Panamericana de 
la Salud. 2018. Disponible en: https://iris.paho.org/bitstream/handle/10665.2/34959/9789275320037 spa.pdf?sequence $=5$ \&isAllowed $=\mathrm{y}$

22. Bryant D, Valaitis R, Martin R, Donald F, Morán L, Brousseau L. Enfermería con práctica avanzada: una estrategia para lograr la cobertura universal de salud y el acceso universal a la salud. Rev. Latino-Am. Enfermagem. 2017; 25:1-11.

23. Beltrán-Salazar OA. Cuidado impersonal o cuidado humanizado: ¿una decisión tomada por enfermeras? Modelo de reloj de arena. Investigación y Educación en Enfermería. 2016; 34 (3):444-455.

24. Fernández-Salazar S, Ramos-Morcillo A, Leal-Costa C, García-González J, Hernández-Méndez S, Ruzafa-Martínez M. Competencia en Práctica Basada en la Evidencia y factores asociados en las enfermeras de Atención Primaria en España. Atención Primaria [Internet]. 2021;53(7):102050. Disponible en: https://dx.doi. org/10.1016\%2Fj.aprim.2021.102050

25. Reyes-Vergara K. Rol Interdependiente de Enfermería en un Modelo de Atención Centrado en el Paciente (MACP): revisión Integrativa. [Tesis en Internet]. 2019 [citado: 2021, junio] Universidad Nacional de Colombia Sede Bogotá Facultad de Enfermería.

26. Laverde-Contreras OL, Carvajal-Carrascal G, Jaimes-Carvajal N, Escobar-Ramórez MC, Arroyo-Marles LP. Rol independiente y ambiente de la práctica de enfermería. Rev Cubana Enferm [Internet]. 2017 [citado 9 Jun 2021]; 33(4):[aprox. 0 p.]. Disponible en: http://www.revenfermeria.sld.cu/index.php/enf/article/view/1013

27. De Arco-Canoles OdelC, Suarez-Calle ZK. Rol de los profesionales de enfermería en el sistema de salud colombiano. Univ. Salud. 2018; 20(2):171-182. DOI: http://dx.doi.org/10.22267/rus.182002.121

28. Cassiani SH, Aguirre-Boza F, Hoyos MC, Barreto MF, Peña LM, Mackay MC, et al. Competencies for training advanced practice nurses in primary health care. Acta Paulista de Enfermagem. 2018; 31(6):572-584. https://doi.org/10.1590/1982-0194201800080

29. Gómez-Salgado J, Navarro-Abal Y, López-López MJ, Romero-Martín M, Climent-Rodríguez J. Engagement, passion and meaning of work as modulating variables in nursing: A theoretical analysis. International Journal of Environmental Research and Public Health, 2019; 16(1). https://doi.org/10.3390/ijerph16010108

30. Organización Mundial da Salud - OMS) Declaración de Astaná. [Internet] 2018. Retrieved from: https:// www.who.int/docs/default-source/primary-health/declaration/gcphc-declaration-sp.pdf

31. Allamde-Cusso R, Macías-Seda J, Porcel-Gálvez AM. La relación enfermera-paciente: identidad histórica, metodológica y terapéutica en los cuidados de enfermería. Cultura de Los Cuidados. 2019; 23(55):78. https:// doi.org/10.14198/cuid.2019.55.08

32. Galavote HS, Zandonade E, Garcia AC, Freitas P, Seidl H, Contarato P, et al. The nurse's work in primary health care. Esc. Anna Nery [Internet]. 2016 Mar [cited 2021 Feb 04]; 20(1):90-98. Available from: http://www. scielo.br/scielo.php?script=sci arttext\&pid=S1414-81452016000100090\&lng=en

33. Torrents R, Ricart M, Ferreiro M, López A, Renedo L, Lleixà M, Ferré C. Ansiedad en los cuidados: una mirada desde el modelo de Mishel. Index de Enfermería. 2013; 22(1-2):60-64. https://dx.doi.org/10.4321/S1132$\underline{12962013000100013}$

34. Barbiani R, Nora CR, Schaefer. Prácticas de enfermería en el contexto de la atención primaria de salud: una revisión del alcance. Rev. Latino-Am. Enfermagem [Internet]. 2016 [consultado el 4 de febrero de 2021]; 24: e2721. Disponible en: https://doi.org/10.1590/1518-8345.0880.2721 